\title{
Le moi minimaliste dans Mes mauvaises pensées
}

\section{The minimalist ego in Mes mauvaises pensées}

\author{
VICTORIA FERRETY \\ Universidad de Cádiz \\ victoria.ferrety@uca.es
}

\begin{abstract}
Sometimes named by literary critic as a descendant of Marguerite Duras, the conception of her work seeks above all to represent the ambivalence of a fragmented intimate self, as well as the sexual and amorous between-two. In relation to his writing, in Mes mauvaises pensées Nina Bouraoui reveals through a process of deconstruction/construction, a writing of the self that condenses processes that recall on certain occasions the nature of texts with component Minimalist. If the presence of the double is permanent in the narrative, the use of the minimalist writing will allow to formulate the presence of the autobiographical self, as well as its relationship to the world.
\end{abstract}

\section{Key-words}

Minimalist writing, fragment, double, psiconalisis.

\begin{abstract}
Resumen
Varias veces nombrada por la crítica literaria como descendiente de Marguerite Duras, la concepción de su obra busca sobre todo representar la ambivalencia de un yo íntimo fragmentado, así como el punto intermedio sexual y amoroso. En relación a su escritura, en Mes mauvaises pensées, Nina Bouraoui revela a través de un proceso de deconstrucción/ construcción, una escritura del yo que condensa procesos que recuerdan en ciertas ocasiones la naturaleza de los textos con componentes Minimalista. Si la presencia del doble es permanente en la narrativa, el uso de la escritura minimalista permitirá formular la presencia del yo autobiográfico, así como su relación con el mundo.
\end{abstract}

\section{Palabras clave}

Escritura minimalista, fragmento, doble, psicoanálisis. 


\section{Introduction}

Au-delà d'une diverse cartographie des modes d'écrire présente chez les romanciers du XXI ${ }^{e}$ siècle, l'écriture de Nina Bouraoui a pour particularité une totale discontinuité entre l'acte de penser et l'extériorité d'un discours autobiographique qui sied au cœur même de ses œuvres. Dans la plupart des études déjà effectuées qui portent actuellement sur 16 romans ${ }^{1}$, c'est surtout autour de la quête identitaire, ainsi que l'entre-deux sexuel et amoureux que s'instaure l'écriture novatrice de cette jeune romancière. Nommée parfois par la critique littéraire comme une descendante de Marguerite Duras, la conception de son œuvre cherche surtout à représenter l'ambivalence d'un moi intime morcelé.

En rapport à Mes mauvaises pensées qui se présente sous forme d'autofiction, et pour laquelle elle obtint le prix Renaudot en 2005, Nina Bouraoui laisse entrevoir à travers un processus de déconstruction/construction, une écriture du moi qui condense des procédés qui rappellent en certaines occasions la nature des textes fragmentés, une des variables du concept minimaliste. En effet, Nina Bouraoui reprend quelques éléments appartenant à l'écriture fragmentaire quand elle décrit des scènes ou des mini-séquences qui sont juxtaposées sans transition ou bien, élabore un type de narration qui s'en tient à ce qui est perceptible et se déroule à travers des actions et une mise en scène qui sont réduites au minimum. Par ailleurs, un autre point légitime dans l'écriture de ce roman est que la narratrice module la réduction $\mathrm{du}$ discours autobiographique à sa plus simple expression le rendant difficile d'accès, en l'éloignant du modèle canonique du roman traditionnel. Paradoxalement, si la réductibilité de son discours manifeste la difficulté à formuler l'acte de pensée, "le choix du petit [lui permet également] d'exprimer une double postulation: déchiffrer l'expérience individuelle afin de la recouvrer contre les universels mensongers" (Adorno, 2003: 350) qui vont à l'encontre de la liberté personnelle d'un tout un chacun. Si le discours minimaliste a affaire à

[une] représentation non contradictoire d'au moins deux événements asynchrones et se rapportant l'un à l'autre sans se présupposer ou s'impliquer logiquement (Bedrane \& Revaz \&Viegnes, 2012: 13).

il ouvre la voie à "la présence obsédante d'[une] narration - certes éclatée, déstructurée, voire déniée, parce qu'impossible" (ibid.) qui indique à son tour combien l'ouverture du moi se dérobe au moment même où il se prétend être réalisable et continu. En fait, grâce au "fragment [qui lui] permet de renouveler sans cesse (1) la posture du narrateur, (2) l'clat bouleversant de l'attaque"(Quignard, 1986: 54), Mes mauvaises pensées contestent les illusions de l'immuable qui peuvent être inlassablement remises en question en laissant instantanément libre-cours à la fois, à une écriture méditative et cognitive qui réfléchit à l'état

1 Son dernier roman Tous les hommes désirent naturellement savoir est sorti en 2018. 
antagonisme existant entre la pensée jaillissante du moi et une instance énonciative qui est fondamentalement fondée

\begin{abstract}
sous la pulsion d'une forte surdétermination, [en empruntant] des formes diverses (désordres spatio-temporels, achronologies, représentation fragmentée des personnages, scission du "je" narratif) pour exprimer des sens multiples et variés de la rupture (Paterson, 1993: 20).
\end{abstract}

Nina Bouraoui met 'l'accent [...] sur la subjectivité et l'intimisme, [qui sont] abordés avec gravité et profondeur, dans un parcours intériorisé, [en] rattachant ce mode [d'écriture] à une exploration intime du réel" (Huglo \& Leprik, 2010: 31,32) car il confère à son œuvre une dimension tensionnelle renouvelée dans une démarche poétique et esthétique qui renvoie à un ordre caché d'un moi libérateur lorsqu'elle déclare : “il y a moi, sous l'écriture de mon livre, il y a l'écriture de ma thérapie, sous le Je. [...]" (Bouraoui, 2007: 36). En se livrant au hasard de sa pensée fragmentée, et se laissant aller à l'indécidable et à l'imprévisible de l'écriture thérapeutique, elle essaie surtout de formuler ce qui est informulable:

Je crois à un livre qui informerait son auteur sur lui, au fur et à mesure de son élaboration, ce serait un livre surnaturel [...] (Bouraoui, 2007: 252)

Empêchée par une prédisposition narrative pour exprimer les pensées indicibles, irréalisables, absentes de par leurs formes et sens originels, elle montre combien l'écriture symbolise le lieu commun où malheureusement la réalité l'emporte sur le reste:

Souvent je préfère lire au lieu d'écrire, parce que la lecture m'arrache au réel, tandis que l'écriture - mon écriture - m'oblige à m'y tenir au plus près. (Bouraoui, 2007: 50).

Dans la relation de cette double intermittence discursive décrite dans Mes mauvaises pensées, nous verrons donc comment la narratrice se sert de l'écriture minimaliste pour formuler la présence du moi autobiographique, puis nous tenterons d'analyser de quelle manière, celle-ci, influence son rapport au monde.

\title{
2. Le moi autobiographique
}

Si une des spécificités de ce roman est la prétention en matière autobiographique qu'il propose, la principale caractéristique semble pourtant être que Nina Bouraoui délègue à son double qui est la narratrice de ses écrits, l'autofiction d'une confession psychanalytique. En d'autre termes, l'histoire d'une reconnaissance et d'une œuvre par le moyen d'une écriture en soi qui vise à relater ses propres souvenirs, avec une série de portraits morcelés qui sont parachevés dans une conscience réelle-fictive créant l'imaginé-vécu. D'un autre côté, on 
distingue la présence d'un journal intime, catalyseur du moi quotidien, et celui-ci peut-on dire, joue un rôle important puisqu'il interfère dans la narration discontinue de ce roman en trahissant la nature rétrospective du pacte autobiographique de Lejeune. Un passage illustrant cette pause temporelle au sein même de son discours thérapeutique est interrompue par la présence de son journal intime lorsque la narratrice manifeste:

Mon père préfère mes romans à mon journal, il déteste cette forme, de la vie annotée, répertoriée, cette somme amoureuse; il dit qu'il ne faut pas arrêter le temps [...] (Bouraoui, 2007: 17).

En légitimant cette croyance de continuité temporelle qu'elle réalise fermement durant la rédaction du journal intime, elle montre sa totale méconnaissance envers l'existence du moi fragmenté car comme le précise Pla:

Etroitement lié au concept de discontinuité, le journal intime est un exemple d'écriture fragmentaire qui semble s'annoncer comme la seule expression littéraire capable de refléter un monde qui, lui aussi, est devenu fragmenté et décomposé. (Pla, 2000: 195).

À son insu, le rôle attribué à la "post-écriture" du journal intime va donner naissance à la réapparition de la structure fragmentaire lorsqu'elle se débarrasse du souvenir des épisodes surannés en excluant sciemment le passé chronologique dépeint dans le journal intime pour ne se limiter qu'à reproduire l'expérience vécue et ressentie durant l'analyse en cours:

Je pourrais écrire le roman de ma thérapie, je pourrais écrire sur nos rendez-vous, ce serait une histoire d'amour, ou une histoire de haine, je ne sais pas encore [...] Je sais que je suis en train de vous donner un livre, je sais que je suis en en train de contrôler mon langage et mes gestes (Bouraoui, 2007: 32).

En d'autres termes, si l'écriture thérapeutique devient implicitement le lieu par excellence de la discontinuité narrative c'est surtout parce qu'elle favorise l'expression et le passage du moi refoulé, inconscient de l'être. Reléguée ainsi à un second plan, l'écriture du journal intime va être en quelque sorte étouffée par le surgissement d'un autre type d'écriture considérée plus impulsive et passionnelle de la part de la narratrice car elle prétend seulement mettre en place les réminiscences et la reconstruction d'un moi intelligible revêtant toutes les formes réelles et imaginaires qui parcourent à la fois distincts espaces temporels et types d'enchâssements de la pensée:

'Ton imagination te joue des tours.' Il faut de l'imagination pour vivre, pour avoir des mauvaises pensées, pour écrire sur soi, puisqu'on ne se connaît jamais vraiment; il faut de l'imagination pour se raconter, pour trouver la réponse à la question 'Qui suis-je?' (Bouraoui, 2007: 59). 
Par la formulation de ces deux types d'écriture discontinue, on remarque des fins bien différentes, car en effet, dans la première purement formelle, Bouraoui utilise le fragment pour concrétiser dans un lieu de la mémoire la consolidation de son identité, car

le journal intime ou personnel contribue, en tant que lieu de mémoire, à un rattachement historique, à la construction de repères temporels, dont l'un des avantages et l'un des buts recherchés sont la consolidation d'une identité [...] (Allam \& Lejeune, 1996: 254).

Tandis que dans la seconde, en minimisant et s'écartant de l'écriture fragmentée du journal intime elle surdimensionne par l'aphasie des mots non verbalisés l'expression spontanée de la mémoire comme unique source fiable de connaissance de soi. Lorsqu'elle énumère durant son analyse une série de phrases qui appartiennent au discours oral, placées côte à côte, et ne paraissent avoir aucune relation entre elles, elle provoque ainsi l'inintelligibilité de la lecture et le refus du commentaire et de la complicité de l'altérité omniprésente:

J'ai acheté une robe hier. - Les orchidées ont une durée de vie de trente ans. - Je vais aller voir mes parents. Je veux du pain - Le gâteau vient de chez Picard. [...] C'est quoi ta bague? Pourquoi as-tu changé de parfum? [...] Il y a quelque chose dans l'air on étouffe. [...] On passe au salon? (Bouraoui, 2007: 89).

Faites d'un vocabulaire simple, à travers ces phrases courtes où le langage métaphorique est absent, la narratrice projette avant tout une écriture du non-dit, cryptographiée qui ne retient pas le superflu du détail inutile qui sert à identifier le reste. Elle ouvre et ferme la propriété des mots en fonction de ses diverses pensées et parfois même, éclaire sur ses besoins du langage qui semblent insatiables et vivants:

Avant j'écrivais dans ma tête, puis j'ai eu les mots, des spirales de mots, je m'en étouffais, je m'en nourrissais (2007: 12) [...] J'ai des milliers de mots dans ma tete, des milliers de petits organismes vivants (Bouraoui, 2007: 26).

Pour justifier l'apparition de l'écriture, cette avant-scène où l'organisation textuelle reste encore chimérique pour exprimer l'existence d'un moi défaillant et manquant qui semble coupé du poids du langage, elle met en évidence la complexité d'un langage illimité qui lui, ne serait soumis à aucune règle et serait connecté à une vie organique indépendante lui permettant d'agir librement sur le langage à venir et la manifestation du moi, car

la connaissance de soi transcende la conscience de soi. Elle la dépasse dans tous les sens, elle risque de la distendre et de l'adultérer. La conscience de soi ne peut que s'affirmer telle quelle. Elle n'a rien à dire d'elle-même. La connaissance de soi au contraire suppose l'expérience dans toute sa complexité, dans son opacité, dans son impureté [...] (Gusdorf, 1948: VII-VIII). 
Par la reconstruction de l'expérience autobiographique où les mots germent à profusion pour ériger une pensée et un rapport légitime de soi à soi, dans un passage du roman on constate à nouveau l'insistance de la narratrice pour personnifier avant tout l'écriture comme une conscience physiologique semblable à un organe du corps. Celle-ci, curieusement s'organise ouvertement autour et surtout par l'intrusion de l'écriture minimaliste dans la construction du roman :

Moi je suis dans une écriture blanche, j'en ai conscience, c'est une conscience organique, je pense à mon cerveau, à ses matières molles, aux milliers de ramifications qui me font écrire, qui me font douter [...] (Bouraoui, 2007: 21-22).

À travers les propos de Roland Barthes qui a instauré dans Le degré zéro de l'écriture (1953), l'expression d'écriture blanche pour désigner un minimalisme stylistique dont l'œuvre inaugurale est celle de L'Étranger (1942), la narratrice exalte une volonté d'écrire un langage nouveau qui la rapprocherait de l'absence énonciative présente dans le roman de Camus "libérée de toute servitude à un ordre marqué du langage" (Barthes, 1972: 55).

Extériorisant ainsi la portée d'une écriture blanche autobiographique dans "je suis dans [...]", elle évoque pourtant à la suite, un autre procédé littéraire appartenant au mouvement surréaliste qui lui, par contre, semble renforcer l'impossibilité énonciative de laquelle elle essaie de se soustraire:

[...] j'ai peur d'altérer ce mécanisme-là, la main libre, la main qui raconte. J'ai peur de tout perdre, j'ai peur de placer mon sujet dans le verbe, j'ai peur de déstructurer mon langage (Bouraoui, 2007: 22).

Assurément, dans ce passage la narratrice manifeste son vif effroi en employant en italique les caractères de "la main libre" car ils matérialisent le principe même de l'écriture automatique promulguée par André Breton qui est celle qui permet de libérer l'expression inconsciente en vue de se connecter avec le moi intérieur. En effectuant cette dichotomie entre écriture blanche et écriture automatique qui sont deux techniques d'écritures reconnues, elle expose sa nette préférence en tant qu'écrivain envers la première. En effet, seule l'écriture blanche arrive à stimuler sa confiance car elle se positionne comme une sorte de garde-fou de la conscience, tandis que l'autre par contre, permet le risque et l'émergence du double engendré par le pouvoir de la peur inconsciente qui peut à tout moment venir l'éloigner de la conscience, de la volonté contrôlée, et même à son insu modifier la façon d'écrire en rendant irréalisable sa mission autobiographique. En fin de compte, l'écriture automatique symbolise la part inconnue que la narratrice refoule en permanence par la menace qu'elle suppose car elle peut susciter "Un rêve, un demi-songe, [...] un état où [elle] ne contrôlerait plus rien" de la même manière que "A., le philosophe qui poignarda sa femme [...]” (Bouraoui, 2007: 11). 
Si elle est donc consciente du piège encouru pour mener à bien son projet d'écriture, et malgré les nombreuses précautions prises elle se heurte pourtant à des difficultés qui elles, ne dépendent plus d'elle: "Je ne peux pas écrire sur le rien, les mots s'effacent, chaque fois parce que je m'efface." (Bouraoui, 2007: 48). Par rapport à ce passage dans lequel la narratrice remarque ce double effacement, on peut constater combien la prégnance de l'écriture blanche au lieu de lui faciliter le projet d'écriture semble plutôt difficulter le développement du moi autobiographique. À ce sujet Ballans explique que "[...] celui qui écrit de cette écriture blanche, impersonnelle, tend à une écriture de formulation mathématique, donc sans auteur: le sujet disparaît pour n'être plus que le scribe qui rend compte (2007: 20).

Faisant le constat de la mort de l'écriture créative et de l'abstraction du sujet narrant postulé dans le mouvement postmoderniste, Nina Bouraoui découvre à son détriment que les effets nocifs produits par ce type d'écriture la conduisent vers une destruction de sens et de déshumanisation de son moi intime:

Il y a de cela dans l'écriture, je dois rendre des comptes, je dois écrire ce que je vois, c'est ma façon d'habiter l'existence, c'est ma façon de fermer ma peau, pour effacer mes mauvaises pensées (Bouraoui, 2007: 79).

L'effacement ${ }^{2}$, ainsi projeté, en rendant manifeste la mécanique énonciative et les rouages des actes de la parole, souligne surtout comment le processus de décomposition de la matière textuelle ainsi que la désarticulation de la prise de parole du "je" s'opposant à la réalisation de son dessein autobiographique.

Saisissant donc que tous les mots ne servent pas à retranscrire son roman car ils peuvent parfois faire disparaître le sujet-écrivant, alarmée du pouvoir que peuvent exercer certains procédés littéraires qu'elle met en place lors de son écriture inexpérimentée, Nina Bouraoui s'aperçoit que les obstacles qui sont liés à son état d'âme ${ }^{3}$ sont aussi provoqués par une impuissance ressentie face au déploiement des règles de la rhétorique:

Elles m'ont invitée [...] parce que je suis triste, parce que je ne sais plus écrire; je pense que j'ai appris seule à écrire des livres, que cet apprentissage est sauvage et qu'il peut s'annuler vite, qu'il peut se perdre comme on peut perdre un chemin ou perdre sa mémoire (Bouraoui, 2007: 39).

2 L'œuvre de Samuel Beckett, peut être à certains égards considérée comme un fragment désarticulé par rapport à l'effacement et la négation du "je".

3 En faisant référence à un autre livre dont le titre est semblable à celui de Nina Bouraoui, et qui se présente sous forme de fragments, dans la maxime 159 des Mauvaises pensées choisies de Nietzsche on retrouve une maxime qui pourrait définir ce que la narratrice considère comme état d'âme: "Les pensées sont les ombres de nos sentiments - toujours obscures, plus vides, plus simples que ceux-ci.”(Nietzsche, 2000: 355). 
En conséquence, elle découvre que la perte de l'écriture implique la déconstruction de l'œuvre autobiographique à cause du manque de mots qui ne veulent plus jaillir par manque de méconnaissance du moi l'empêchant par conséquent d'écrire :

J'ai perdu mon écriture pendant trois ans, j'ai repris mon rôle buvard auprès de ma famille, j'ai entendu un livre que je ne pouvais pas retranscrire, j'ai reçu un livre de paroles que je ne pouvais pas convertir, je me tenais à côté de la littérature, je regardais les trains passer. [...] J'étais en faillite de moi-même (Bouraoui, 2007: 29-30).

En fait, l'incapacité de l'écriture autobiographique ressemble plutôt à un châtiment subi qu'elle expérimente dans une profonde douleur car elle la dirige vers une sorte d'acte de restauration émotionnelle:

L'écriture est aussi une prison, je dois la justifier, je dois la réparer, je dois la supplier quand elle ne vient pas, quand elle est mauvaise (Bouraoui, 2007: 35).

Arme à double tranchant, l'acte d'écriture inexperte se profile proche du balbutiement d'un nouveau né et semble être vécu en fonction des éléments extérieurs qui ressemblent parfois à un miracle: "Mon écriture est revenue, je suis née une seconde fois" (Bouraoui, 2007: 30).

Le paradoxe de l'écriture blanche qui s'auto-efface alors qu'elle apparaît, en se voyant elle-même réfléchie dans la ruine de sa propre impuissance gagne en sens car elle se manifeste à travers sa propre néantisation. En accueillant donc l'indicible, Nina Bouraoui nous invite aussi à voir ce que le langage veut bien montrer.

Par ailleurs, la connaissance de certaines techniques narratives, qu'elle analyse au moment même où s'initie l'acte d'écriture rend manifeste que la construction du roman autobiographe s'élabore aussi à partir d'une mémoire intermittente qui dépend à son tour, d'une écriture discontinue et indépendante de la propre volonté du moi intime. Comme le souligne Gusdorf:

Le commencement des écritures du moi correspond toujours à une crise de la personnalité; l'identité personnelle est mise en question, elle fait question; le sujet découvre qu'il vivait dans le malentendu. Le repli dans le domaine de l'intimité répond à la rupture d'un contrat social fixant le signalement de l'individu selon l'ordre d'apparences usuelles dont l'intéressé s'aperçoit brusquement qu'elles sont abusives et fondées (Gusdorf, 1990: 23).

On remarque que l'écriture minimaliste joue un rôle fondamental dans la reconstruction de la pensée car elle met en question la relation entre le moi et l'autre reconfigurée par l'expérience humaine, et à travers la transformation d'une recherche identitaire propulsée par l'écriture. Interrogeant donc les propres limites du moi minimaliste, la narratrice reprend la 
symbolique de la spirale lorsqu'elle dit “J'ai eu des spirales de mot” (Bouraoui, 2007: 12), pour formuler le véritable désir de sa pensée nouvelle libérée de toute contrainte affligeante du passé. À cet effet, Roland Barthes, a défini la dualité de cette figure ainsi:

La spirale règle la dialectique de l'ancien et du nouveau; grâce à elle, nous ne sommes pas contraints à penser: tout est dit, ou: rien n'a été dit, mais plutôt rien n'est premier et cependant tout est nouveau (Barthes, 1992: 199).

Si dans "la spirale de mots" Nina Bouraoui, reflète la projection vers l'infini du langage qu'elle essaye d'atteindre explicitement avec “[des] mots nouveaux" mais surtout "[des] mots préférés" (Bouraoui, 2007: 14) qui lui permettraient de bâtir une œuvre autobiographique d'un moi fait sur mesure, qui absorberait la dialectique de l'ancien et du nouveau pour créer le langage de son propre univers, elle expose aussi explicitement sa difficulté de combler son rapport au monde pour matérialiser le moi car “une identité n'est jamais donnée, reçue ou atteinte, non, seul s'endure le processus interminable, indéfiniment phantasmatique, de l'identification" (Derrida, 1996: 37).

Pour ainsi dire, l'appropriation du moi constitue toujours un procédé de déconstruction car il met en rapport l'insaisissable du langage à la recherche d'une réunification avec la connaissance de soi au monde.

\section{Le rapport au monde du moi minimaliste}

Dans l'essai de Voleur de mots (1985) Michel Schneider fait une analyse sur les pensées en expliquant que ce sont des écrits fragmentés. D'une part, il explique que le fragment renvoie à une forme littéraire d'une extrême brièveté qui fait appel à la prose. D'autre part, la forme du fragment questionne le fractionnement de la mémoire et de la pensée puisqu'elle est réduite à une temporalité existentielle. En nous basant sur les connaissances techniques d'écriture de Nina Bouraoui qu'elle confirme dans Mes Mauvaises pensées, tout porte à croire que le choix du titre de son roman autobiographique n'est pas un fait isolé. En effet, si son titre peut faire allusion aux Pensées de Blaise Pascal, la particularité de l'écriture fragmentaire est qu'elle "se démarque des genres en les pratiquant tous afin d'échapper au piège de la totalitét" en adoptant un no man's land romanesque qui va favoriser la narratrice à recréer son rapport au monde. Autrement dit, de la même manière que la projection de ce type d'écriture échappe aux frontières du récit, elle incite à questionner les limites entre l'identité et la différence, entre le moi et l'altérité, entre l'intime et l'inconnu quand elle entreprend son entreprise autobiographique en employant des constructions indépendantes qui s'enchaînent

4 Voir en ligne: <litterature.ens-lyon.fr/littérature/dossiers/poesie/ecriture-fragmentaires/pierre-garrigues>, consulté le 20 avril 2019. 
les unes aux autres et qui défilent au bon gré de sa mémoire avec un rapport au monde où le moi est lui-même, jugé fragmenté. En effet, comme le mentionne Anne-Marie Clément:

\begin{abstract}
L'écrivain qui choisit le discontinu peut vouloir rendre compte d'un univers fragmenté et hétérogène, de l'expérience discontinue du réel découlant d'une perception parcellaire ou inordonnée du monde, de la fragmentation des savoirs, d'un sujet divisé dont la voix ne correspond plus à un flux continu. C'est en somme l'idée de totalité qui échappe au sujet, un sujet pour qui aucun savoir ne vient légitimer une connaissance du tout, un sujet pour qui l'interprétation du monde n'est possible qu'en termes d'interprétation partielle et singulière du monde (Clément, 2005: 42).
\end{abstract}

Au moment même où Nina Bouraoui réitère "je suis revenue de moi et tout est parti de moi [...]" (Bouraoui, 2007: 16), elle montre tout d'abord, la volonté de contrôle du moi autobiographique en adoptant certaines formes de l'écriture blanche avec le dessein de créer la sienne, puis le rejet de la structure inconsciente de l'écriture automatique qui interroge en permanence l'incertitude d'un moi minimaliste difficile à cerner, car ce dernier en effet semble susceptible d'ouvrir la voie à un sentiment particulier de l'effrayant perçu comme quelque chose d'indéfinissable et non localisable s'emparant d'elle lors de la retranscription de son autobiographie. Ainsi, outre la présence stabilisante de son journal intime signalé dans certains passages du roman, les efforts de la narratrice pour développer une dimension narrative du moi autobiographique, montrent à contre-courant, une parole qui elle, par contre paraît assurément être sous le contrôle de l'inconscient puisqu'elle se construit à l'intérieur d'une perspective psycho-analytique, c'est-à-dire, un discours clos fermé sur lui-même. En effet, au début du roman, la narratrice sous forme monologale s'adresse à Madame C., sa thérapeute pour lui raconter :

Je viens vous voir parce que j'ai des mauvaises pensées. Mon âme se dévore, je suis assiégée. Je porte quelqu'un à l'intérieur de ma tête, quelqu'un qui ne serait plus moi ou qui serait un moi que j'aurais longtemps tenu, longtemps étouffé (Bouraoui, 2007: 11).

À travers le discours psychanalytique décrit par l'apparition du trouble mental souffert par la narratrice constatant "je me considère comme une personne malade" (Bouraoui, 2007: 14), Nina Bouraoui intercale un discours qui a pour rôle de venir transgresser les règles de son projet autobiographique car la justification même de l'inconscient présuppose l'inclusion du non-sens dans le discours. De même que l'immersion de l'inconscient altère toute objectivité au discours oral, elle renvoie également et donne naissance à une sorte d'alter égo, image reflet du morcellement de la structure minimaliste, car

le fragment est défini comme le morceau d'une chose brisée, en éclats, et par extension le terme désigne une œuvre incomplète morcelée. Il y a, comme l'origine étymologique le confirme, brisure, et l'on pourrait parler de bris de clôture de texte. La fragmentation est d'abord une violence subie, une désagrégation intolérable (Montandon, 1992: 77). 
En fait, l'atemporalité et le non-sens exhibés par le discours psychanalytique correspondent à l'enjeu proposé par le fragmentaire et l'écriture minimaliste car ils sont toujours “suspects de mixité" (Susini-Anastopoulos, 1997: 49) et donc difficiles à catégoriser. Il ressort que ces deux approches ont un point en commun car elles représentent la pensée de l'inconciliation qui vient révéler ce qui échappe. Ainsi, là où la narration échoue dans sa fonction symbolisante, l'expérience du langage ainsi que l'acte analytique prennent place: “alors que je déploie un livre, le livre rêvé, qui ne s'écrit pas mais qui se dit” (Bouraoui, 2007: 16).

Si bien l'écriture minimaliste recouvre la totalité de son œuvre, l'insertion du discours psychanalytique projette la reconstruction de l'altérité du moi perçu et conçu doublement par la narratrice, miroir du premier, qui le déplace, qui le dépasse et le reprend. Rappelons que:

Par le double, l'unité devient divisible, le double représentant l'altérité passant par une altération du un qui fait deux, faisant notamment jouer le rapport entre une image interne et une image externe, c'est-à-dire le rapport entre le moi idéal et l'Idéal du moi. (Besançon \& Osganian, 2003: 201-217).

En choisissant donc la voie de l'analyse de ses pensées autobiographiques pour restaurer son rapport à l'autre, la narratrice construit en sus du discours minimaliste un discours de l'inconscient, où le moi laisse parler librement le ça, grâce au discours psychanalytique redevable à l'association libre de la cure freudienne, car

on peut faire endosser au double non seulement ce contenu qui heurte la critique du
moi; on peut lui attribuer aussi toutes les possibilités avortées de forger notre destin
auxquelles le fantasme veut s'accrocher encore, et toutes les aspirations du moi qui
n'ont pu aboutir par suite de circonstances défavorables, de même que toutes les dé-
cisions réprimées de la volonté, qui ont suscité l'illusion du libre arbitre.(Freud, 1985:
238).

Devant les diverses manifestations du moi contrairement à Barthes qui formule "Au fond, c'est pour multiplier à moi-même, ce plaisir que j'ai opté (provisoirement) pour l'écriture discontinue." (Barthes, 1981: 174), celles-ci sont vécues autrement par la narratrice car la propre analyse du moi correspond aux moyens d'échapper à l'angoisse de la multiplication d'un assaillant redoutable, d'une “identité du deux-en-un" (Mattéi, 2006: 40) engendrée par des phrases courtes qui interférent à l'intérieur du récit autobiographique:

Je suis visitée par mes propres informations (Bouraoui, 2007: 32-33) [...] J'aimerais me défaire de mon cerveau (ibid., 11) [...] Je suis hors de mon sujet, hors de moi (ibid., 30) [...] Je suis ce corps, je ne suis plus ce corps (ibid., 34) [...] Je ne sais plus qui je suis (ibid., 15).

Autrement dit, de la même façon qu'elle justifie une autoréflexion, mais aussi une réflexion indirecte sur la nature du moi minimaliste "[qui] est une écriture de restitution"(Bou- 
raoui, 2007: 136), la pulsion scopique psychanalytique permet surtout de mettre en scène la dialectique entre regarder et être regardé, en particulier lors du développement de la phase du miroir qu'elle manifeste dans "Je n'ose plus me regarder dans le miroir [...]" (Bouraoui, 2007: 12).

Cette confrontation du moi et de l'inconscient, du moi de la narratrice face à son œuvre révèle précisément que cette insuffisance signifiante du langage est influencée par la plasticité de la parole indicible qui ne se montre qu'à travers le silence du double. Par conséquent, la fonction du double, en même temps qu'elle pointe du doigt la confusion de la figure inconsciente du moi de la narratrice est aussi celle, qui l'empêche d'atteindre la voie de l'individuation. Par rapport à la fonction de l'inconscient, pour C.G. Jung, cette voie de l'individuation joue un rôle primordial car elle permet

[de] tendre à devenir un être réellement individuel et, dans la mesure où nous entendons par individualité la forme de notre unicité la plus intime, notre unicité dernière et irrévocable, il s'agit de la réalisation de son Soi, dans ce qu'il y a de plus personnel et de plus rebelle à toute comparaison. On pourrait donc traduire le mot d' "individuation" par "réalisation de soi-même", "réalisation de son Soi" (Jung, 1964: 115).

Devant la mise en épreuve de retranscrire une confrontation réelle pour retrouver son moi légitime et son rapport à l'autre:

L'inconscient exige de la part de l'individu un effort de conscience et un point de vue de conscience ferme, capable de s'opposer à l'inconscient et de parlementer avec lui. (Jung, 1964: 204).

Par le choix de l'écriture blanche qui est en soi "un arrangement avec le réel" (Bouraoui, 2007: 14), les mots psychanalytiques opèrent une double rupture avec le sujet autobiographique: d'une part, ils montrent la déficience d'un discours lacunaire exposant les limites du langage, d'autre part, ils prouvent l'existence de "mots qui viennent à l'intérieur d'euxmêmes, du corps caverneux et obscur" (Bouraoui, 2007: 59).

A travers la signification de "ces mots à l'intérieur d'eux-mêmes", où se situe la structure inconsciente du moi psychanalytique qu'il faut déchiffrer pour comprendre la matière invisible, impalpable et effrayante du moi oppresseur, l'image hallucinatoire correspond au contrepoint symbolique de la rupture avec la réalité du mot présent et du cadre spatio-temporel du roman.

Hors du commun romanesque, la représentation implique surtout la peur refoulée de la narratrice et de son double appréhendé dans de nombreux passages du récit qu'elle essaie de canaliser à travers le langage et le récit minimaliste. Si dans un premier temps, le double exprime une figure instable qui recoupe plusieurs réalités, plusieurs moi parcellaires à l'instar de l'autre, la construction psychanalytique sous-tend une tension résiduelle qui ramène la 
figure du double vers une représentation du conflit psychique. Figure de la limite et du paradoxe, le double pour Nina Bouraoui constitue donc l'interface d'un croisement romanesque entre un discours minimaliste et psychanalytique qui joue des cartes similaires avec la réduction des composantes de la diégèse.

Malgré que leurs fins semblent totalement divergentes car les enjeux diffèrent dans la structure romanesque, leur point commun favorise autant le bouleversement de la vision du monde que l'instrumentalisation du moi par le langage. En relation à l'écriture minimaliste qui remet sans cesse en question la réalité du langage, l'apparition de l'image psychanalytique greffée au mot correspondrait donc à la métaphore du langage refoulé, inconséquent donc irréel faisant œuvre d'une fragmentation du moi réinvesti autrement, en vue d'être interprété par la suite. Cette corrélation que l'on retrouve entre ces deux discours s'inscrit bien dans l'optique des "minimalistes" français car "le récit se présente comme une représentation. [...]" (Schoots, 1977: 57-58). Par ailleurs, en déréalisant le réel, l’œuvre de Nina Bouraoui montre combien:

l'originalité de l'écriture minimaliste réside donc dans sa façon de redécouvrir le récit tout en mettant en cause la représentation de la réalité par le langage [...] D'une part, l'écriture minimaliste poursuit donc le travail subversif de l'avant-garde minimaliste, d'autre part elle redécouvre les éléments narratifs traditionnels rejetés par cette même avant-garde (Schoots, 1977: 57-58).

Durant cette recherche d'autonomisation du moi effectuée sous divers angles narratifs, si la structure du discours minimaliste l'écarte de l'inconscient exprimé par "la main libre", on peut avancer que le discours psychanalytique de manière isolée et presque compulsivement lui sert de tremplin pour exprimer son moi et son rapport au monde. Ainsi, peut-on comprendre qu'en correspondance avec la projection de son moi minimaliste-inconscient hors-limite, Nina Bouraoui façonne une écriture qui se tient en dehors de l'édifice spatio-temporel, avec un mouvement qui n'annonce ni son début ni sa fin ${ }^{5}$ et s'en tient uniquement à “entrer et sortir, non pas commencer ni finir" (Deleuze \& Guattari, 1980: 36-37).

Au-delà de l'hybridation du discours et l'emploi de certaines figures de style qu'elle

5 Dans son essai sur l'écriture fragmentée intitulé Roland Barthes, vers le neutre, Bernard Comment mentionne aussi cette impossibilité de début et fin dans 1'écriture minimaliste: "Il apparait surtout que le fragment a pour caractéristique d'être achevé mais pour exigence, pas facilement compatible, de ne pas se clore, ou mieux: de ne jamais assener sa clôture. J'anticipe ici pour suggérer que le fragment, comme écriture de l'instant, offrira une solution possible à cette sorte de double-bind (achever sans conclure) par la suppression de durée dont il se voudra le lieu. Dès lors, 'plus de montée' possible, plus de pointe. Ni de Dans son essai sur l'écriture fragmentée intitulé Roland Barthes, vers le neutre, Bernard Comment mentionne aussi cette impossibilité de début et fin dans l'écriture minimaliste: "Il apparaît surtout que le fragment a pour caractéristique d'être achevé mais pour exigence, pas facilement compatible, de ne pas se clore, ou mieux: de ne jamais assener sa clôture. J'anticipe ici pour suggérer que le fragment, comme écriture de l'instant, offrira une solution possible à cette sorte de double-bind (achever sans conclure) par la suppression de durée dont il se voudra le lieu. Dès lors, 'plus de montée' possible, plus de pointe. Ni de commencement à proprement parler, ni de fin, puisque tous deux confondus dans le geste même de saisie." (Comment, 2002: 173). 
emploie dans son roman autobiographique, l'autofiction va lui servir à se disperser indéfiniment en élaborant la fuite et le retour de son moi obsessionnel parcellé mais, également transmettre les pertes inconscientes de la mémoire que ponctue la vacuité de son discours minimaliste qui est:

la réplique de tout brusquement serve de l'individualisation exacerbée de soi. Symbole insistant dans le deuil natif où tout baigne. Minuscule catastrophe, minuscule épave, et minuscule solitude (Quignard, 1986: 45).

Certes, si pour l'expression de ses mauvaises pensées Nina Bouraoui se prête à une narration oscillatoire car "le roman se divise en un objet à la fois regardant et regardé, littéraire et métalittéraire” (Dallenbach, 1972: 56), qu'elle exprime à travers un tissu textuel décousu qui répondrait à la fois à la perspective minimaliste et psychanalytique, c'est surtout pour montrer que la nature du moi reste fondamentalement chaotique malgré les efforts:

En un sens, toute écriture est écriture du moi. Mais, le plus souvent, ce moi qui fait œuvre d'écriture parle d'autre chose ; la littérature du moi commence par l'usage privé et réfléchi d'une écriture qui, au lieu de s'accrocher à n'importe quoi, à la manière des paroles qui s'envolent dans le courant des jours, s'enracine dans la présence de soi à soi qu'elle s'efforce de rendre intelligible à elle-même. (Gusdorf, 1990: 124).

La nature du moi intime morcelé replié sur lui-même exprime notamment l'impossibilité de la narratrice pour concevoir son œuvre autobiographique car "la partie de la personnalité qui refoule l'autre a pris en main les leviers de commande et dirige". (Mauron, 1986: 33) En formalisant ses mauvaises pensées, par la présence du double qui est omnisciente le long du récit, elle formule spécialement l'incapacité de son rapport au monde. C'est pourquoi l'œuvre définitive ne semble réalisable qu'à travers le rêve:

Je fais souvent le rêve du livre idéal, qui s'écrirait dans un état d'inconscience, un livre qui dirait le secret de notre famille, un livre surgi de l'hypnose, le livre de la vérité, puisque c'est cela le grand problème: savoir; il faut que je sache (Bouraoui, 2007: 166).

Durant cette quête incessante d'une écriture qui resterait fidèle à ses pensées, ce que Nina Bouraoui fonde principalement c'est la reconnaissance du moi fantasmé car selon Bachelard:

Pour nous connaître doublement en être réel et en être idéalisant, il nous faut écouter nos rêveries. Nous croyons que nos rêveries peuvent être la meilleure école de la "psychologie des profondeurs ${ }^{6 "}$ (Bachelard, 1968: 64).

$6 \quad$ La théorie de la psychologie des profondeurs fut élaborée par Carl Gustav Jung en 1913. 
En définitive, dans cette démarche minimaliste et psychanalytique qui donne lieu à reconstruction de la voix et des pensées de la narratrice, Nina Bouraoui entraîne le lecteur vers un lieu-dit autobiographique commun, un topos qui rassemble son œuvre liminaire, vers une "représentation cyclique qui n'est autre que la consécration d'un moi intime parcellé" se frayant un espace dans le monde. Grâce à la particularité de ce type d'écriture fragmentée, elle nous invite à la suivre, à la penser, à la déchiffrer en même temps que la narratrice sert d'exercice à la création romanesque:

Derrière les mots qui se lisent, comme avant les mots qui s'écrivent, il y a une voix déjà inscrite, non entendue et non parlante, et l'auteur est, auprès de cette voix, à égalité avec le lecteur: tous deux, presque confondus, cherchant à la reconnaître (Blanchot, 1969: 482).

\section{Conclusion}

Le roman de Mes Mauvaises pensées de Nina Bouraoui peut être considéré à maints égards, en plus de la tentative de narrer une histoire autobiographique, une réalisation artistique qui réunit les éléments d'une écriture blanche qui sont mentionnés le long du récit, mais également, une confirmation minimaliste présente dans beaucoup de romans contemporains. En plus des nombreuses pistes se rapportant à l'écriture minimaliste, on remarque également un autre type de discours qui tient à la psychanalyse et qui fonctionne en parallèle de la mise en narration générale du récit minimaliste. Si ce dernier ouvre la voie à la nature exacte des silences et mots inconscients qu'on peut retrouver durant une séance de psychanalyse, il agit aussi en tant que moteur organisateur de la narration pour retranscrire la vraisemblance de l'analyse de la narratrice. Par ailleurs, bien que sa fonction semble détournée de son ensemble romanesque dans une première lecture on décèle qu'elle joue le même rôle que les autres structures émises lors du récit, à savoir l'identification du moi, son exposition et analyse en cours, en d'autres termes la construction/déconstruction du moi en vue d'une éventuelle nouvelle projection. Rejoignant tous les possibles, l'écriture psychanalytique représentée sous cet élan incandescent et pulsionnel du moi semble être la juste mesure pour surplomber l'ensemble créateur de son œuvre qui se résiste insidieusement et contrecarre avec le rythme impromptu d'une écriture minimaliste.

Parmi les efforts et brouillages narratifs et discursifs que l'écriture impose et qu'elle peut assumer car elle rejette une classification particulière du genre, il reste quand même difficile d'imaginer et croire l'acte de l'écriture comme une construction purement ex nihilo, parce que "la création d'un texte implique déjà des choix il n'existe pas de texte nu, ni de degré zéro de l'écriture" (Schaeffer, 1989: 185). Faute d'expérience, peut-être, ou tout simplement clair désir de fourvoyer les limites et normes du récit autobiographique, Nina Bouraoui, expose formellement dans ce roman une exigence discontinue multiforme comparable à celle que produisent succinctement les pensées. 
Les réfléchissant comme un effet miroir, les techniques romanesques de tout ordre servent d'une part, à justifier leurs existences, ainsi qu'à légitimer en permanence le questionnement d'un moi complexe, hors-catégorie littéraire. Si Gusdorf fixe la prise de conscience au moment même "où le sujet prend la parole" (1990a: 41), Bouraoui dans son roman, dans un double jeu, expose cette évolution du moi qu'elle essaie de transgresser par tous les moyens car “dire [et écrire] sa vie, c'est changer sa vie” (1990a: 43). En devenant donc, objet de l'écriture dans ce roman qu'elle édifie en un bloc, où aucun paragraphe n'apparaît, elle alterne une procédure de sens et de formes appelant dans le texte une recherche de rupture, d'inaccomplissement et d'hermétisme volontaires pour montrer que l'enjeu de l'écriture de la pensée sont avant tout un moyen de réflexion aléatoire ${ }^{7}$ intemporel.

Pour finir notre analyse, nous remarquons que de la même manière que pour Julia Kristeva le roman est "un PROCESSUS de mutation. La forme romanesque [...] un jeu, un changement constant, un mouvement vers une finalité déçue, ou, disons en termes actuels, une TRANSFORMATION" (1970: 17).

Les Mauvaises pensées de Nina Bouraoui accomplissent bien la tâche de l'écrivain contemporain lorsqu'elle édifie une œuvre en partant de: "Je rêve d'un livre de transformation" (2007: 22).

\section{Références bibliographiques}

AA.VV. [consulté le 20 avril 2019].<litterature.ens-lyon.fr/littérature/dossiers/poesie/ecriture-fragmentaires/pierre-garrigues $>$.

Adorno, Theodor W. 2003. Minima Moralia. Réflexions sur la vie mutilée. Paris, Payot.

Allam, Malik et LeJeune Philippe. 1996. Journaux intimes: une sociologie de l'écriture personnelle. Paris, L'Harmattan.

Bachelard, Gaston. 1968. La poétique de la rêverie. Paris, Presses universitaires de France.

Ballans, 2007. L'écriture blanche: un effet du démenti pervers. Paris, L'Harmattan, coll. "Psychanalyse et civilisations."

BARThes, Roland. 1981. Le grain de la voix. Paris, Seuil.

Barthes, Roland. 1992. L'Obvie et l'Obtus. Paris, Seuil.

Bedrane, Sabrinelle, Revaz, Françoise et Viegnes, Michel. 2012. Le récit minimal. Du minime au minimalisme. Paris, Presses Sorbonne Nouvelle.

Besançon, Karine et Osganian Valérie. 2003. "Rencontre avec un jeune homme: le double comme figure résolutive" in La clinique lacanienne, $\mathrm{n}^{\circ} 6,201-217$.

7 On retrouve cette mention d'aléatoirité dans le fragment 472 des Pensées de Blaise Pascal: "J'écrirai ici mes pensées sans ordre et non pas peut-être dans une confusion sans dessein. C'est le véritable ordre et qui marquera toujours mon objet par le désordre même" (Pascal, 2004: 335). 
Anales de Filología Francesa, n. ${ }^{\circ}$ 27, 2019

Victoria FERRETY

Blaise, Pascal. 2004. Pensées. Paris, Gallimard, coll. "Folio”.

Blanchot, Maurice. 1969. L'entretien infini. Paris, Gallimard.

Bouraoui, Nina. 2007. Mes Mauvaises pensées. Paris, Folio.

Clément, Anne-Marie. 2005. Formes et sens de la discontinuité dans la prose narrative québécoise contemporaine. Québec, Université Laval.

Comment, Bernard. 2002. Roland Barthes, vers le neutre. Paris, Christian Bourgois Éditeur.

Dallenbach, Lucien. 1972. Le livre et ses miroirs dans l'œuvre romanesque de Michel Butor. Paris, Minard, Archives des lettres modernes, VIII, nº 135.

Deleuze, Gilles et Guattari, Félix. 1980. "Rhizome” dans Mille plateaux. Paris, Éditions de Minuit.

DerridA, Jacques. 1996. Le monolinguisme de l'autre ou la prothèse d'origine. Paris, Galilée.

Freud, Sigmond. 1985. "L'inquiétante étrangeté" in L'inquiétante étrangeté et autres essais. Paris, Gallimard.

GuSDORF, Georges. 1948. La découverte de soi. Paris, PUF.

Gusdorf, Georges. 1990. Lignes de vie 1 : Les écritures du moi. Paris, Odile Jacob.

Huglo, Marie-Pascale et LePrIK, Kimberley. 2010. "Narrativités minimalistes contemporaines. Toussaint, Tremblay, Turcotte" in Littérature québécoise. Voix et images, 106, automne, 31-32.

KRISTEVA, Julia. 1970. Le texte du roman. Approche sémiologique d'une structure discursive transformationnelle. Paris, Mouton.

MattéI, Jean-François. 2006. L'énigme de la pensée. Chemins de pensée. Nice, Ovadia.

Mauron, Charles. 1986. L'inconscient dans l'œuvre et la vie de Racine. Paris, Champion-Slatkine.

Montaudon, Alain. 1992. Les formes brèves. Paris, Hachette.

Nietzsche, Friedrich. 2000. Mauvaises pensées choisies. Paris, Gallimard, coll. "Tel”.

Paterson, Janet. 1993. Moments postmodernes dans le roman québécois. Ottawa, Presses de l'Université.

Pla, Xavier. 2000. “La feinte autobiographique. L'écriture de Josep Pla, entre autobiographie et autofiction." Perceptions et réalisations du moi. Clermont-Ferrand, Presses Universitaires.

Quignard, Pascal. 1986. Une gêne technique à l'égard des fragments. Paris, Fata Morgana.

SCHNEIDER, Michel. 2011. Voleur de mots. Essai sur le plagiat, la psychanalyse et la pensée. Paris, Gallimard. 
Anales de Filología Francesa, n. ${ }^{\circ}$ 27, 2019

LE MOI MINIMALISTE DANS MES MAUVAISES PENSÉES

Scноот, Fieke. 1997. "Passer en douce à la douane”. L'écriture minimaliste de Minuit : Deville, Echenoz, Redonnet et Toussaint. Amsterdam, Rodopi, coll. "Faux titre".

Shaeffer, Jean-Marie. 1989. Qu'est-ce qu'un genre littéraire? Paris, Editions du Seuil.

Susini-Anastopoulos, Françoise. 1997. L'écriture fragmentaire. Paris, PUF. 